\title{
Metal oxide semiconductor gas sensor self-test using Fourier-based impedance spectroscopy
}

\author{
M. Schüler, T. Sauerwald, and A. Schütze \\ Laboratory for Measurement Technology, Department of Mechatronics, Saarland University, \\ Saarbrücken, Germany \\ Correspondence to: M. Schüler (m.schueler@lmt.uni-saarland.de)
}

Received: 31 July 2014 - Revised: 4 September 2014 - Accepted: 6 September 2014 - Published: 25 September 2014

\begin{abstract}
For the self-test of semiconductor gas sensors, we combine two multi-signal processes: temperaturecycled operation (TCO) and electrical impedance spectroscopy (EIS). This combination allows one to discriminate between irreversible changes of the sensor, i.e., changes caused by poisoning, as well as changes in the gas atmosphere. To integrate EIS and TCO, impedance spectra should be acquired in a very short time period, in which the sensor can be considered time invariant, i.e., milliseconds or less. For this purpose we developed a Fourier-based high-speed, low-cost impedance spectroscope. It provides a binary excitation signal through an FPGA (field programable gate array), which also acquires the data. To determine impedance spectra, it uses the ETFE (empirical transfer function estimate) method, which calculates the impedance by evaluating the Fourier transformations of current and voltage. With this approach an impedance spectrum over the range from $61 \mathrm{kHz}$ to $100 \mathrm{MHz}$ is acquired in ca. $16 \mu \mathrm{s}$.

We carried out TCO-EIS measurements with this spectroscope and a commercial impedance analyzer (Agilent 4294A), with a temperature cycle consisting of six equidistant temperature steps between 200 and $450{ }^{\circ} \mathrm{C}$, with lengths of $30 \mathrm{~s}\left(200^{\circ} \mathrm{C}\right)$ and $18 \mathrm{~s}$ (all others). Discrimination of carbon monoxide $(\mathrm{CO})$ and methane $\left(\mathrm{CH}_{4}\right)$ is possible by LDA (linear discriminant analysis) using either TCO or EIS data, thus enabling a validation of results by comparison of both methods.
\end{abstract}

\section{Introduction}

Metal oxide semiconductor (MOS) gas sensors are highly sensitive to a broad range of reducing and oxidizing gases, and they are available at relatively low cost. Their operation is based on resistance measurements of a sensitive layer, which in most cases consists of a granular metal oxide. The resistance of this layer is strongly determined by the adsorption of oxygen, which creates a depletion region at the metal oxide surface leading to an energy barrier between grains (Tricoli et al., 2010; Morrison, 1982; Kohl, 1989).

The interaction of adsorbed oxygen and reducing or oxidizing gases on the grain surfaces depends strongly on temperature and shows different behavior, depending on the gases and temperatures (Clifford and Tuma, 1982, 1983; Morrison, 1987). Temperature is usually controlled by an integrated heater, e.g., a microstructured platinum resistor on the substrate.
By variation of temperature, it is possible to obtain a virtual multi-sensor or virtual sensor array, i.e., to evaluate the sensor resistance at different temperatures and thereby gain selectivity, in a manner similar to the use of multi-sensory arrays (Stetter and Penrose, 2002; Schütze et al., 2004). This method, which we denote as temperature-cycled operation (TCO), can be used to increase the selectivity and sensitivity of metal oxide gas sensors considerably (Heilig et al., 1997; Lee and Reedy, 1999).

Another way to increase the selectivity is the measurement of the complex sensor impedance by electrical impedance spectroscopy (EIS). One of the underlying effects is the change in capacity at the grain boundaries, which is caused by gas, primarily oxygen. The dielectric properties of the chemical species present in the sensing layer also influence the capacitance properties of the sensor layer - their measurement by EIS can thus increase selectivity (Weimar and Göpel, 1998; Bârsan and Weimar, 2003). 


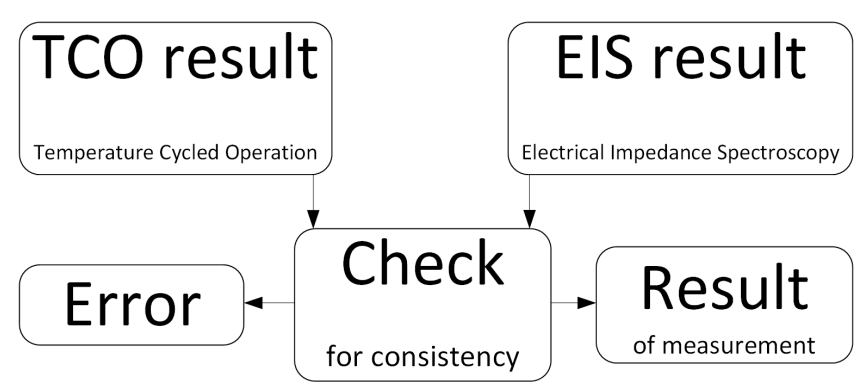

Figure 1. Scheme of the sensor self-test strategy (cf. Schüler et al., 2014).

Besides selectivity, long-term sensor stability is a challenge for the use of SC gas sensors, especially in safetycritical applications. A variety of factors influence this stability, including structural and phase transformations, poisoning, degradation of contacts and heaters, bulk diffusion, sensor design, humidity, temperature changes and interference effects (Korotcenkov and Cho, 2011). By increasing selectivity, TCO - combined with an optimized signal processing (Fricke et al., 2008) - addresses especially the influence of interference gases and changes in humidity, as well as temperature changes (provided the actual sensor temperature is controlled, not just heater voltage). The combination of TCO and EIS can increase the reliability of MOS gas sensors further, acquiring data which may give additional information on the sensor properties. These data not only reflect poisoning, but also may contain information about structural and phase properties, contacts and heater state (although the heater is usually isolated from the heater electrodes, heater properties may affect the measured data by capacitive coupling), bulk diffusion and humidity (Bârsan and Weimar, 2003). These different properties affect the acquired data in different ways, and the generalization of the method described here requires further research. This paper is confined to the study of poisoning induced by HMDSO (hexamethyldisiloxane), a compound which is present as a solvent in many polymers and causes significant changes in the properties of MOS gas sensors. Previous works have shown that both TCO and EIS can be used to discriminate different gases by multivariate analysis (Conrad et al., 2007). By checking the consistency of results acquired with both methods, the reliability of the measurement can be increased (Reimann et al., 2008). This approach is illustrated in Fig. 1.

To perform EIS and TCO measurements simultaneously, impedance spectra should be acquired in a very short time period (milliseconds or less) during which the sensor temperature (and other properties) can be considered constant, i.e., the sensor as a time-invariant system. For this purpose we developed a Fourier-based high-speed, low-cost impedance spectroscope (Schüler et al., 2014).

\section{Impedance measurement}

The complex impedance $Z(\omega)$ can be defined as follows (Barsoukov and Macdonald, 2005):

$$
Z(j \omega)=|Z| \exp (j \theta)=\frac{F\{v(t)\}}{F\{i(t)\}} .
$$

Here, $F\{v(t)\}$ denotes the Fourier transform of the voltage, and $F\{i(t)\}$ denotes the Fourier transform of the current.

The measurement of impedance can be carried out either in the time domain, which requires a transformation of the measured values to the frequency domain, or it can be carried out in the frequency domain, e.g., by setting amplitude and phase of a driving current and measuring the phase shift and amplitude of the resulting voltage drop across the device under test (DuT). Most commercial high-frequency impedance analyzers work in the frequency domain - this approach promises good accuracy and does not require sophisticated signal processing (Barsoukov and Macdonald, 2005; Agilent 4294A). On the other hand, the measurements take inherently longer, since only one frequency is measured at a given time. For example, the instrument used for the reference measurements presented here requires at least $3 \mathrm{~ms}$ to measure the impedance at one frequency (Agilent 4294A); a highresolution impedance spectrum with, e.g., 200 tested frequencies requires at least $0.6 \mathrm{~s}$. This duration is even higher if frequencies below $100 \mathrm{kHz}$ are considered or high precision is required. On the other hand, when measurements are carried out in the time domain, a broad range of frequencies can be analyzed simultaneously; thus, measurement times can be greatly reduced. However, determination of the impedance spectrum requires signal processing, e.g., Fourier transformation, to transfer data recorded in the time domain to the frequency domain (Barsoukov and Macdonald, 2005).

To achieve a compact measurement system with acquisition times (well) below $1 \mathrm{~ms}$, we built an impedance spectroscope which carries out the measurement in the time domain and transforms it into the frequency domain using FFT (fast Fourier transform), in order to calculate the impedance. This method is known as ETFE (empirical transfer function estimate) and is widely used in system identification to estimate transfer functions of LTI (linear time invariant) systems (Ljung and Glad, 1994). In a general form, the ETFE approach can be written as follows (Ljung and Glad, 1994):

$G_{\mathrm{S}}(\omega)=\frac{Y_{\mathrm{S}}(\omega)}{U_{\mathrm{S}}(\omega)}$.

$G_{\mathrm{S}}(\omega)$ is the transfer function of a system, $Y_{\mathrm{S}}(\omega)$ is the output in the frequency domain, and $U_{\mathrm{S}}(\omega)$ is the corresponding input. We can consider the impedance of a sensor as a transfer function describing the relationship between voltage and current across the sensor:

$$
Z(\omega)=\frac{U(\omega)}{I(\omega)}=\frac{U_{\text {Sensor }}(\omega)}{U_{\mathrm{ref}}(\omega) / R_{\mathrm{ref}}} .
$$




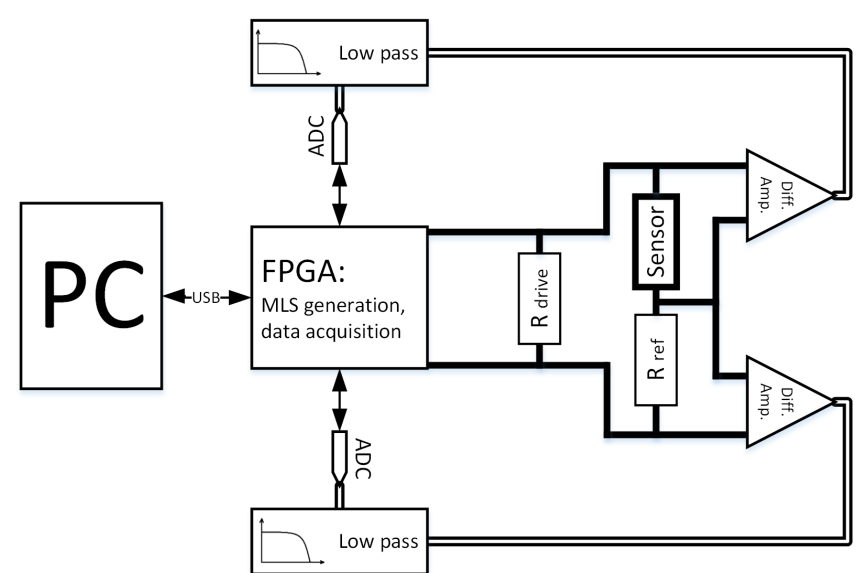

Figure 2. Hardware setup of the FoBIS measurement system (cf. Schüler et al., 2014).

In Eq. (3), $Z(\omega)$ is the impedance, $U(\omega)$ resp. $U_{\text {Sensor }}(\omega)$ the voltage, and $I(\omega)$ the current. $I(\omega)$ is not set or directly measured, but determined from the voltage $U_{\text {ref }}(\omega)$ across a resistor of known impedance $\left(R_{\text {ref }}\right)$.

Figure 2 shows the corresponding hardware setup: a binary signal of broad frequency range is generated by a fieldprogrammable gate array (FPGA) and transmitted using the BLVDS_25 differential signaling standard, which generates a current of defined value. This current causes a voltage drop of $\pm 1.2 \mathrm{~V}$ across the resistor $R_{\text {drive }}(75 \Omega)$, and thereby also across the voltage divider consisting of the sensor and $R_{\text {ref }}$ which is connected in parallel (Reimann et al., 2008). Thus, due to the much higher impedance of the voltage divider compared to $R_{\text {drive }}$, a voltage of $\pm 1.2 \mathrm{~V}$ is applied across the voltage divider to drive the sensor. The branches of this voltage divider are connected to high-speed ADCs (analog-to-digital converters; model ADS62P49EVM, Texas Instruments) via differential amplifiers (AD8130, Analog Devices), which enable a robust signal transmission, as well as low-pass filters, which eliminate frequencies above ca. $100 \mathrm{MHz}$. We use an MLBS (maximum length binary sequence) excitation signal with a length of $16.384 \mu \mathrm{s}$, which is sampled at a frequency of $250 \mathrm{MHz}$. The bandwidth of such a signal ranges from $61 \mathrm{kHz}$ to $125 \mathrm{MHz}$ (Schüler et al., 2014). However, since frequencies above $100 \mathrm{MHz}$ are removed in order to prevent aliasing effects, the bandwidth of the measurement system ranges from $61 \mathrm{kHz}$ to $100 \mathrm{MHz}$. (Schüler et al., 2014).

The introduced measurement method is denoted as FoBIS (Fourier-based impedance spectroscopy). Figure 3 shows measurements of test impedances carried out with the FoBIS system on the one hand, and with the commercial highprecision impedance analyzer Agilent 4294A on the other hand. Obviously, the FoBIS system cannot compete in terms of absolute accuracy. Due to parasitic capacitances, the signal intensity decreases at higher frequency and thereby the SNR

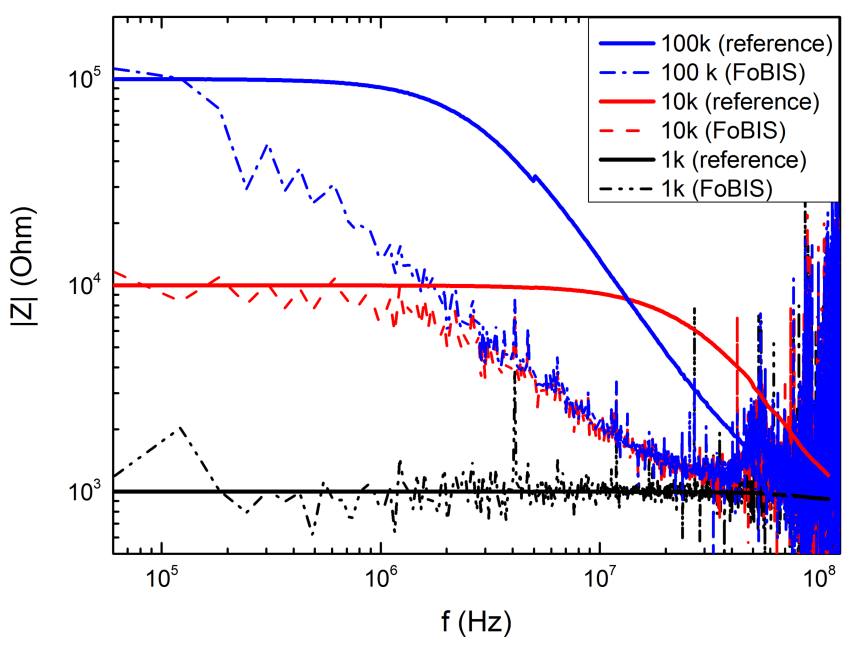

Figure 3. Measurements of test impedances $(1 / 10 / 100 k \Omega$ highprecision resistors with small parasitic capacitances $(<0.4 \mathrm{pF}))$, carried out with a commercial high-precision impedance analyzer (Agilent 4294A) and the FoBIS measurement system.

(signal-to-noise ratio) decreases inherently. On the other hand, the quickness of the measurement principle (acquisition time $\approx 16.4 \mu \mathrm{s}$ ) enables measurements in temperaturecycled operation even for microstructured sensors with thermal time constants in the millisecond range (Elmi et al., 2008).

\section{Experimental}

To evaluate the self-test strategy sketched in Fig. 1, measurements were carried out with the UST Umweltsensortechnik GGS 1330, an $\mathrm{SnO}_{2}$-based sensor sensitive towards a broad range of reducing gases. At first, we characterized the unimpaired sensor. The measurements were carried out in temperature-cycled operation with the Agilent 4294A, and the FoBIS system. To enable temperature-cycled operation with the Agilent 4294A, we implemented the temperature cycle shown in Fig. 4, consisting of six equidistant temperature steps between 200 and $450^{\circ} \mathrm{C}$, with lengths of $30 \mathrm{~s}$ $\left(200^{\circ} \mathrm{C}\right)$ and $18 \mathrm{~s}$ (all others). The impedance analyzer $\mathrm{Ag}$ ilent $4294 \mathrm{~A}$ requires $10.8 \mathrm{~s}$ to record one impedance spectrum with a range from $200 \mathrm{~Hz}$ to $110 \mathrm{MHz}$ with 201 supporting points with logarithmic distribution at the secondhighest precision available ("measurement bandwidth" $=4$ ). Acquisition of the impedance spectra was triggered $5 \mathrm{~s}$ after each temperature change, when the sensor has almost reached a steady state (the impedance relaxation induced by the temperature change is mostly complete, cf. Fig. 7). After these initial characterization measurements, the sensor was exposed to $70 \mathrm{ppm}$ HMDSO for $10 \mathrm{~min}$ in temperaturecycled operation. After this poisoning, the characterization measurements were repeated. Figures 5 and 6 show a section of the characterization measurements carried out before and 


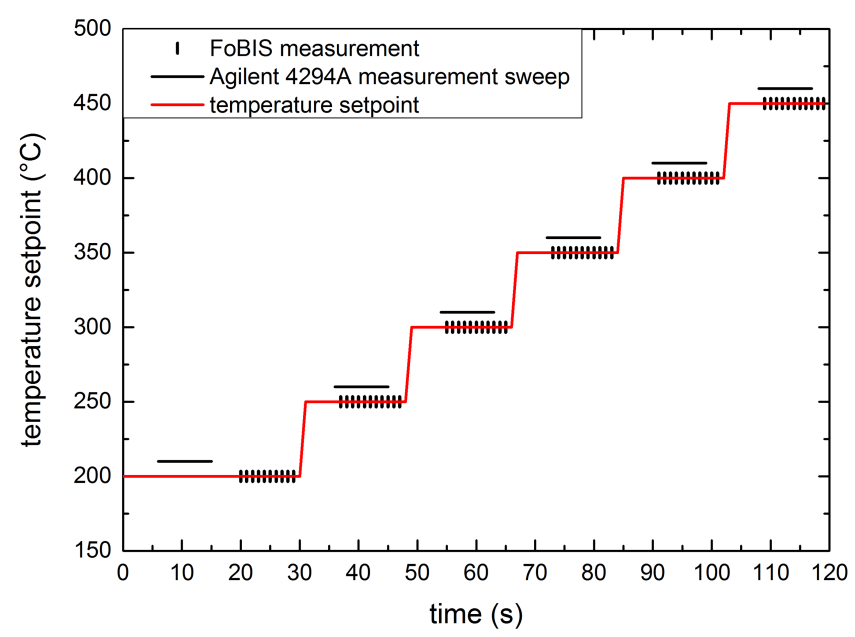

Figure 4. Temperature set points and timing of impedance measurements during temperature cycle.

after poisoning. The presented values are quasi-static sensor responses, i.e., sensor response values which were acquired at defined time/temperature points within the temperature profile. The sensor response is defined as follows:

$S=\frac{\left|Z_{\text {air }}\right|}{\left|Z_{\text {gas }}\right|}-1$,

where $\left|Z_{\text {air }}\right|$ is the magnitude of the impedance in air, acquired for each temperature in the first temperature cycle, and $\left|Z_{\text {gas }}\right|$ is the impedance value at a given time during the measurement.

Figure 5 shows quasi-static sensor responses at $200 \mathrm{~Hz}$ and $62.8 \mathrm{kHz}$, acquired with the Agilent 4294A before and after poisoning. The gas concentrations are shown in the bottom row of Figs. 5 and 6. In this paper, we consider two concentrations each of methane $\left(\mathrm{CH}_{4}: 550\right.$ and $\left.1100 \mathrm{ppm}\right)$ and carbon monoxide (CO: 50 and $100 \mathrm{ppm}$ ); whereas the gas profile also contained hydrogen $\left(\mathrm{H}_{2}: 5\right.$ and $\left.10 \mathrm{ppm}\right)$ and ethanol $\left(\mathrm{C}_{2} \mathrm{H}_{5} \mathrm{OH}: 5\right.$ and $\left.10 \mathrm{ppm}\right)$ (Schüler et al., 2014). All gases were applied in zero air with $50 \%$ rh (relative humidity).

The sensor reactions differ for the different gastemperature combinations in a typical manner $\left(\right.$ at $350^{\circ} \mathrm{C}$, the sensor reactions of $\mathrm{CH}_{4}$ and $\mathrm{CO}$ are comparably high, while at $200^{\circ} \mathrm{C}$, carbon monoxide causes a significantly higher sensor reaction), which enables selective measurements. The quasi-static sensor responses of the unimpaired sensor are very high during the first cycles in presence of methane, especially at high temperatures. This phenomenon might be caused by a reaction of a surface species taking place at higher temperatures in presence of methane, in which the species causing the reaction is being consumed or desorbed. The measurement after poisoning does not exhibit this behavior.

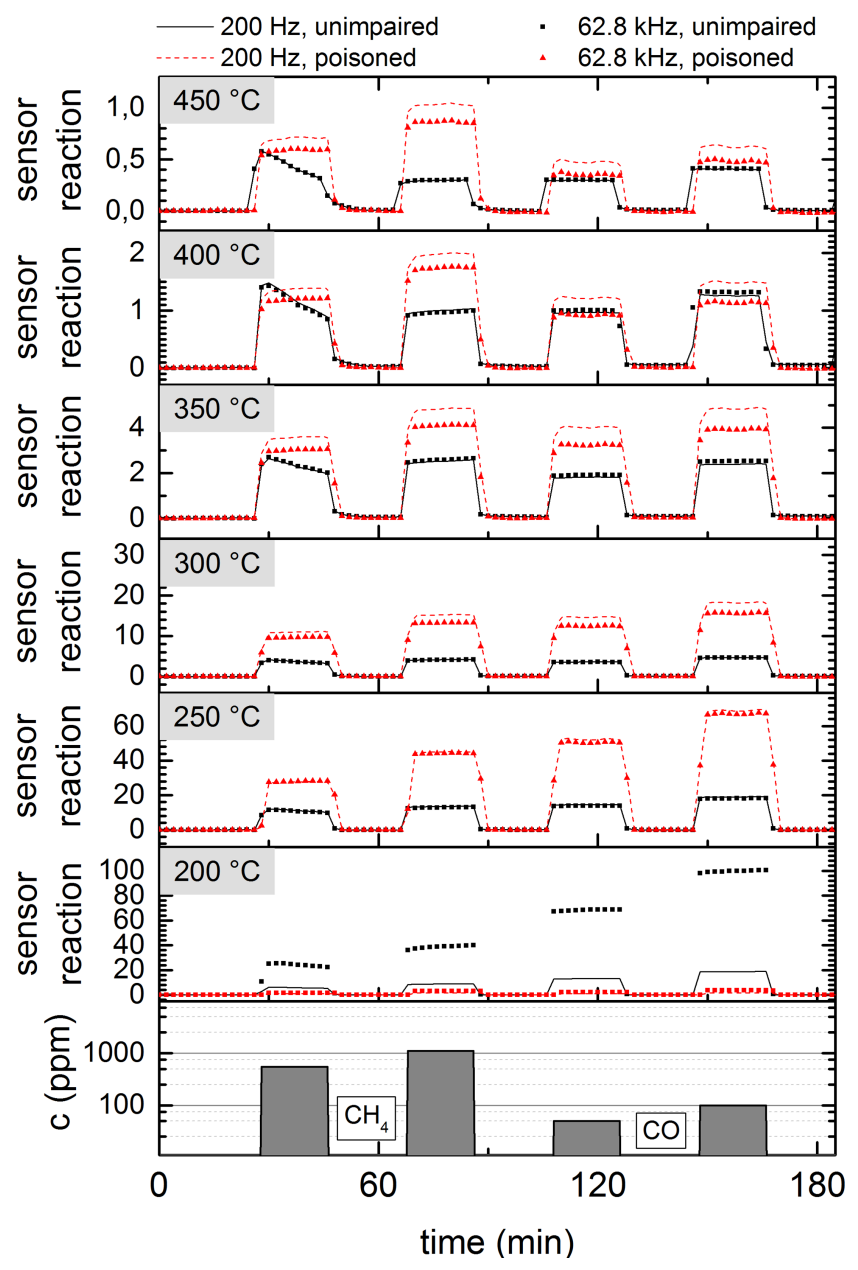

Figure 5. Laboratory measurement, carried out with commercial impedance analyzer Agilent 4294A. The graphs show sensor reactions for each step of the temperature cycle at $200 \mathrm{~Hz}$ and $62.8 \mathrm{kHz}$ with the unimpaired as well as with the poisoned sensor.

For temperatures of $250^{\circ} \mathrm{C}$ and above, the sensor reactions match well for both frequencies, confirming earlier research which has shown that the response at frequencies up to $100 \mathrm{kHz}$ corresponds to the DC resistance which is usually measured (Reimann, 2011). However, at $200^{\circ} \mathrm{C}$, the sensor reaction measured at $62.8 \mathrm{kHz}$ differs significantly from the one measured at $200 \mathrm{~Hz}$. The reason for this difference is a typical resonance effect in the frequency spectrum of the UST GGS 1330, which has been described in Reimann (2011). The frequency, at which this resonance peak appears, depends on the measurement setup and the resistance of the sensor, it shifts towards lower frequencies for high resistances. At $200{ }^{\circ} \mathrm{C}$ the resonance peak is at ca. $100 \mathrm{kHz}$ in air, and the impedance measured is strongly influenced by the resonance. When resistivity drops due to the presence of a reducing gas, the shift of the resonance peak towards higher frequencies is added to the actual drop in resistance, leading to an extraordinarily high sensor response at 


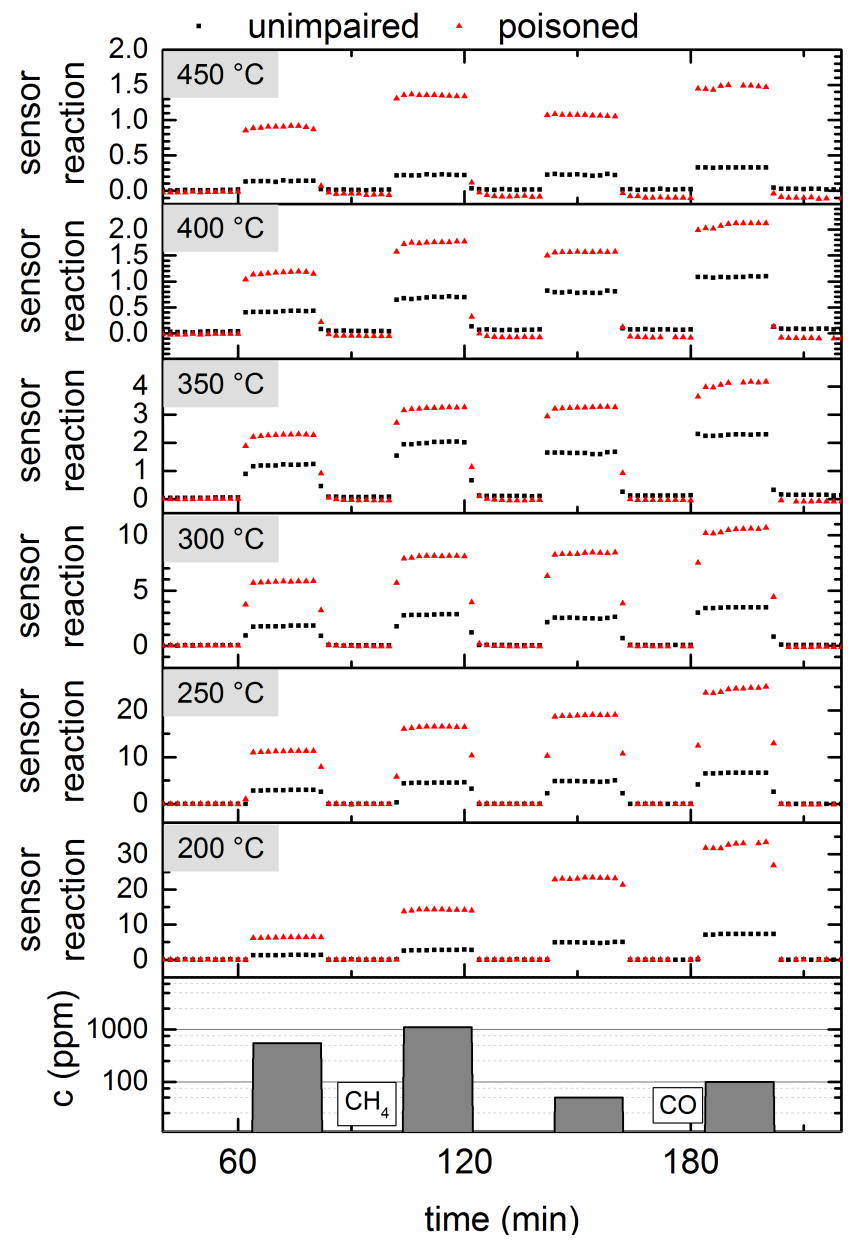

Figure 6. Laboratory measurement, carried out with FoBIS system. The graphs show sensor reactions for each step of the temperature cycle at $61 \mathrm{kHz}$ for the unimpaired as well as for the poisoned sensor.

$62.8 \mathrm{kHz}$. The poisoned sensor has a significantly lowered resistance, at which the resonance peak is shifted towards much higher frequencies, which explains the lower sensor response measured at $200^{\circ} \mathrm{C}$ after poisoning. Overall, the sensor reactions are higher for the poisoned sensor. A decrease in sensitivity is not what one would implicitly expect from a sensor poisoning mechanism which deactivates surface states on the sensor. However, some reflections on the functioning of the sensor give plausibility to the phenomenon: the poisoning reaction of HMDSO with sensor surfaces takes place primarily at the locations which are exhibited most directly to the sensor poison, deactivating these in the first place. The metal oxide layer is contacted at its very bottom by electrodes; thus, the measured resistance is influenced most strongly by the sensor reactions taking place near those electrodes. The poisoning of the upper sensor layers inhibits sensor reactions on the more "electrically insensitive" outer surface and enables the target gas to penetrate to the more "electrically sensitive"

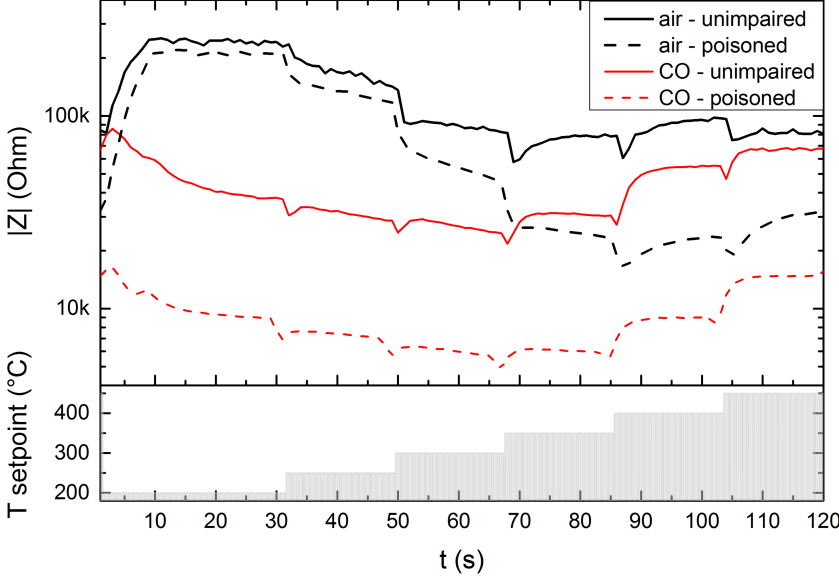

Figure 7. Sensor impedance of UST 1330 at $61 \mathrm{kHz}$, in air (50\%rh, black lines) and in air with $50 \mathrm{ppm}$ CO (red lines) during temperature cycle. The solid lines show the impedance of the unimpaired sensor; the dashed lines show the impedance values of the same sensor after its exposure to $70 \mathrm{ppm}$ HMDSO (hexamethyldisiloxane) during $10 \mathrm{~min}$.

Table 1. Features used for EIS-based LDA.

\begin{tabular}{lll}
\hline Quantity/frequency range (all at $\left.250^{\circ} \mathrm{C}\right)$ & $\begin{array}{l}\text { Mean } \\
\text { value }\end{array}$ & Slope \\
\hline Abs. value of impedance $|Z|(61-183 \mathrm{kHz})$ & & $\mathbf{X}$ \\
Abs. value of impedance $|Z|(456 \mathrm{kHz}-4.6 \mathrm{MHz})$ & $\mathbf{X}$ & $\mathbf{X}$ \\
Abs. value of impedance $|Z|(4.9-6.4 \mathrm{MHz})$ & $\mathbf{X}$ & \\
Abs. value of impedance $|Z|(6.4-8.9 \mathrm{MHz})$ & $\mathbf{X}$ & $\mathbf{X}$ \\
Abs. value of impedance $|Z|(8.9-27.4 \mathrm{MHz})$ & $\mathbf{X}$ & \\
Abs. value of impedance $|Z|(40.8-103 \mathrm{MHz})$ & $\mathbf{X}$ & $\mathbf{X}$ \\
Impedance angle $\Theta(Z)(594 \mathrm{kHz}-5.3 \mathrm{MHz})$ & $\mathbf{X}$ & \\
\hline
\end{tabular}

region in direct vicinity of the electrodes, where the sensor reaction takes place and is measured with high sensitivity.

Figure 6 shows the quasi-static sensor responses of the measurement carried out with the FoBIS system before poisoning. Here, the shown values were calculated by averaging 10 measurements at the end of each temperature step. The reason that 10 data points per temperature step were averaged was to achieve better comparability of the FoBIS measurements with those carried out with the Agilent 4294A, which can only acquire one spectrum per temperature step. Figure 6 shows the sensor responses at $61 \mathrm{kHz}$. At this frequency, the SNR of the FoBIS spectroscope is relatively high and the sensor responses towards all gases are clearly recognizable at all temperatures.

Figure 7 shows sensor impedance values at $61 \mathrm{kHz}$ over the temperature cycle, in pure air with $50 \% \mathrm{rh}$, as well as in presence of $50 \mathrm{ppm} \mathrm{CO}$. The solid lines represent the unimpaired sensor; the dashed lines represent the measurement carried out after poisoning. In air, the poisoning has little influence on the impedance values at lower temperatures, whereas at high temperatures, the impedance of the poisoned 


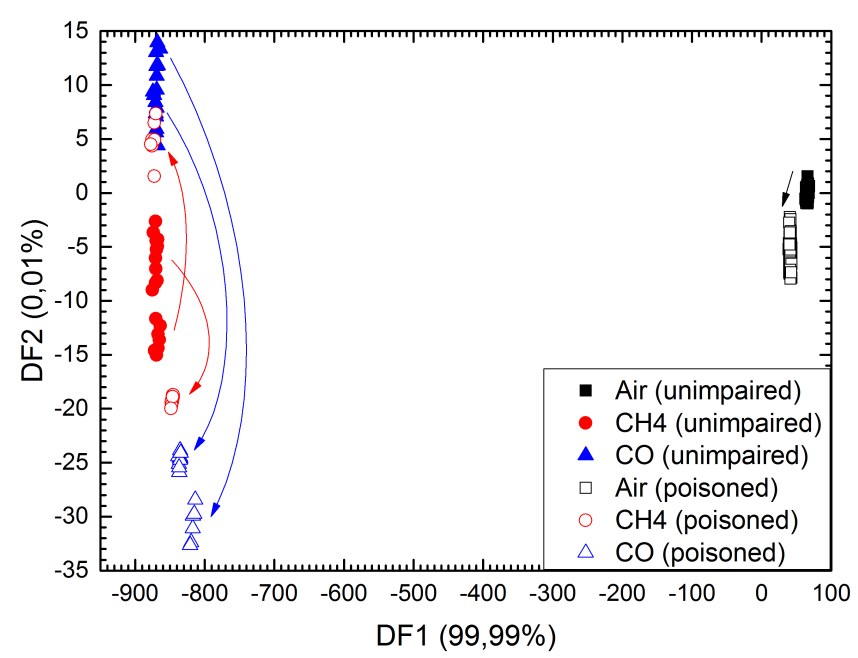

Figure 8. LDA of 10 EIS features (mean values/slopes) for unimpaired (solid symbols) and poisoned (open symbols) sensor, acquired with Agilent 4294A impedance analyzer.

Table 2. Results of leave-one-out cross validation using a kNN classifier $(k=3)$ with Euclidian distance.

\begin{tabular}{llrrr}
\hline \multirow{2}{*}{ Measurement method } & Sensor state & \multicolumn{3}{c}{$\begin{array}{c}\text { Percentage of correct } \\
\text { classifications }\end{array}$} \\
\cline { 3 - 5 } & & Air & $\mathrm{CH}_{4}$ & $\mathrm{CO}$ \\
\hline \multirow{2}{*}{ EIS-IA } & unimpaired & $100 \%$ & $100 \%$ & $100 \%$ \\
& poisoned & $100 \%$ & $62.5 \%$ & $0 \%$ \\
\hline \multirow{2}{*}{ EIS-FoBIS } & unimpaired & $100 \%$ & $81.25 \%$ & $87.5 \%$ \\
& poisoned & $100 \%$ & $0 \%$ & $100 \%$ \\
\hline \multirow{2}{*}{ TCO-IA } & unimpaired & $100 \%$ & $100 \%$ & $100 \%$ \\
& poisoned & $100 \%$ & $100 \%$ & $0 \%$ \\
\hline \multirow{2}{*}{ TCO-FoBIS } & unimpaired & $100 \%$ & $100 \%$ & $100 \%$ \\
& poisoned & $100 \%$ & $50 \%$ & $100 \%$ \\
\hline
\end{tabular}

sensor is strongly reduced compared to the unimpaired sensor. This results in a clearly recognizable change of the shape of the impedance over the temperature profile. In presence of $50 \mathrm{ppm}$ of carbon monoxide, the shape of the impedance over the temperature cycle changes less strongly, whereas there is a strong decrease in the magnitude of the impedance after poisoning, which actually leads to an increase in sensitivity vs. $\mathrm{CO}$ at low sensor temperatures.

\section{Feature extraction, signal processing and results}

To evaluate the measurements and enable a classification of the results, we use LDA (linear discriminant analysis), a supervised algorithm for dimensionality reduction. LDA calculates a linear transformation, which maps a multidimensional feature vector to a vector with $N-1$ dimensions, $N$ being the number of classes to be discriminated (Backhaus et al., 2000). In our case, the classes correspond

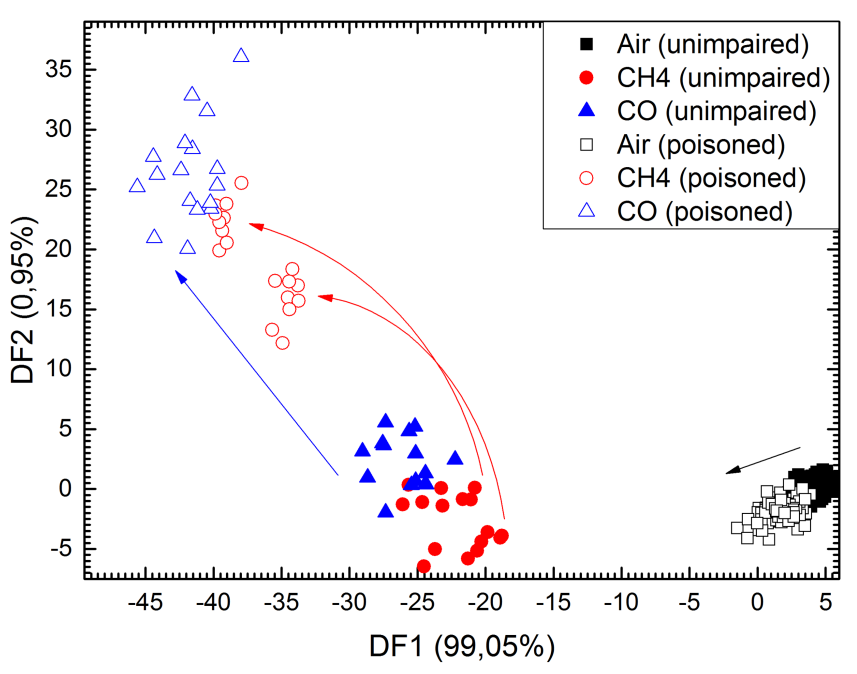

Figure 9. LDA of 10 EIS features (mean values/slopes) for the unimpaired (solid symbols) and the poisoned (open symbols) sensor, acquired with FoBIS impedance spectroscope.

to the different gases to allow discrimination of the different gases independent of the gas concentration. The transformation calculated by the LDA maximizes the distance between data sets from different classes and minimizes the variance (spread) within the single classes. Although LDA reduces the dimensionality, the number of dimensions in the feature vector should be limited to a number much smaller than the number of measurements in order to achieve a stable discrimination, i.e., to prevent overfitting (Luo et al., 2011).

We carried out LDAs with EIS and TCO data, using the commercial impedance analyzer Agilent 4294A and the FoBIS impedance spectroscope. Hereafter, the LDA evaluations are denoted as follows: EIS-IA (EIS data, commercial impedance analyzer), EIS-FoBIS (EIS data, FoBIS impedance spectroscope), TCO-IA (TCO data, commercial impedance analyzer), and TCO-FoBIS (TCO data, FoBIS impedance spectroscope).

The feature vectors for the EIS-IA and EIS-FoBIS LDA were generated by extracting mean values and slopes in selected frequency intervals. Table 1 outlines the features used for these LDAs, which were obtained from the $250^{\circ} \mathrm{C}$ temperature step only. At this temperature, the signal in air is only slightly influenced by the poisoning. Figure 8 shows the EIS-IA LDA. The solid symbols represent measurements carried out with the unimpaired sensor; the open symbols represent the measurements after poisoning. Air is clearly discriminated from both gases by the first discriminant function (DF1), which contains $99.99 \%$ of the discriminatory information. For the unimpaired sensor, $\mathrm{CO}$ and $\mathrm{CH}_{4}$ are discriminated in the second discriminant function (DF2), which contains only $0.01 \%$ of the discriminatory information, and is therefore very sensitive to small changes in the sensor signal. In DF1, there is only a small shift for all groups after 


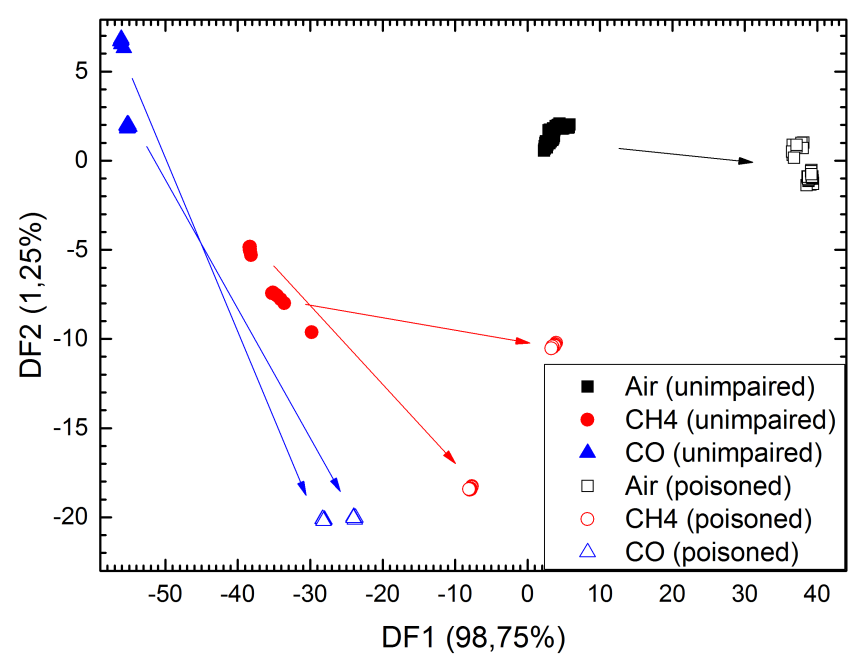

Figure 10. LDA of five TCO features (absolute impedance value differences) for unimpaired (solid symbols) and poisoned (open symbols) sensor, acquired with Agilent 4294A impedance analyzer.

poisoning. Along DF2, however, the shifts are relatively bigger for the gas measurements. These shifts are different for $\mathrm{CO}$ and $\mathrm{CH}_{4}$ : the $\mathrm{CO}$ measurements shift relatively far, those measured at $100 \mathrm{ppm}$ further than the ones taken at $50 \mathrm{ppm}$. The different methane concentrations $(550 \mathrm{ppm} / 1100 \mathrm{ppm})$ shift in crosswise manner. These shifts might result from the long measurement time of the Agilent 4294A, during which the sensor characteristics might change, combined with a higher sensitivity of DF2 to small changes in the sensor signal. We carried out a leave-one-out cross-validation, using a $k$ nearest neighbor $(\mathrm{kNN}, k=3)$ Euclidian distance classifier for the different LDAs (Francois et al., 2011). The results are shown in Table 2. It shows that after poisoning discrimination between $\mathrm{CH}_{4}$ and $\mathrm{CO}$ is no longer possible. However, air is classified correctly even after poisoning, and can thus be discriminated from both gases.

Figure 9 shows the EIS-FoBIS LDA. Similar to the EISIA LDA, this evaluation shows only a small shift in the measurements acquired in pure air caused by poisoning. In the presence of $\mathrm{CO}$ or $\mathrm{CH}_{4}$ the measurements shift along both discriminant functions after poisoning. For the EIS-FoBIS LDA, the shifting directions are rather parallel, unlike in the EIS-IA LDA, where a crosswise shift was observed. In this LDA, the discrimination between $\mathrm{CH}_{4}$ and $\mathrm{CO}$ is imperfect even before poisoning, with $81.25 \%$ and $87.5 \%$, respectively, of measurements classified correctly. After poisoning, all $\mathrm{CH}_{4}$ measurements are classified incorrectly. Although the evaluation results are not completely equivalent for both measurement systems, the differences between the gas measurements of unimpaired and poisoned sensors can be recognized in the LDAs carried out with impedance data from either system, and pure air can be discriminated from gas even after poisoning.

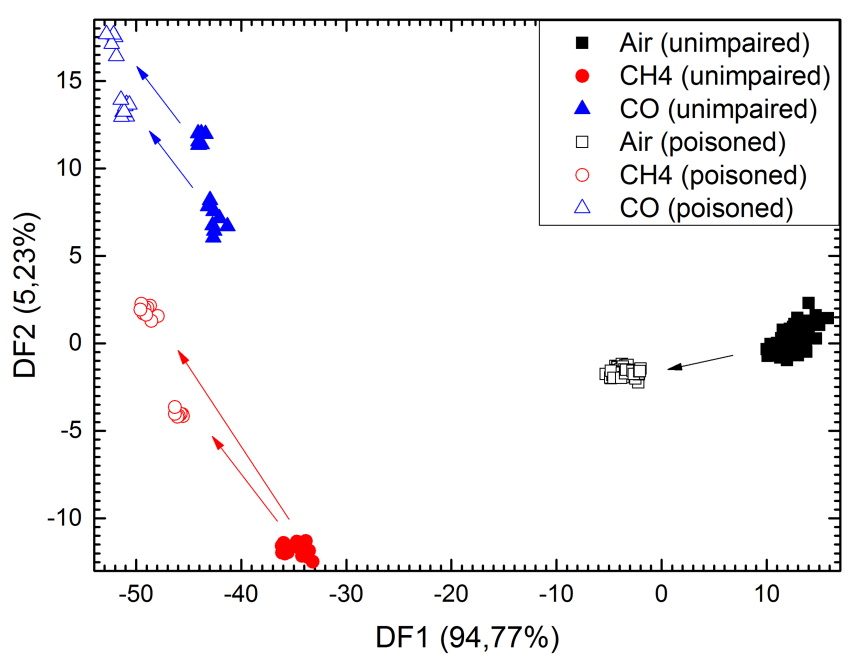

Figure 11. LDA of five TCO features (absolute impedance mean value differences) for unimpaired (solid symbols) and poisoned (open symbols) sensor, acquired with the FoBIS impedance spectroscope.

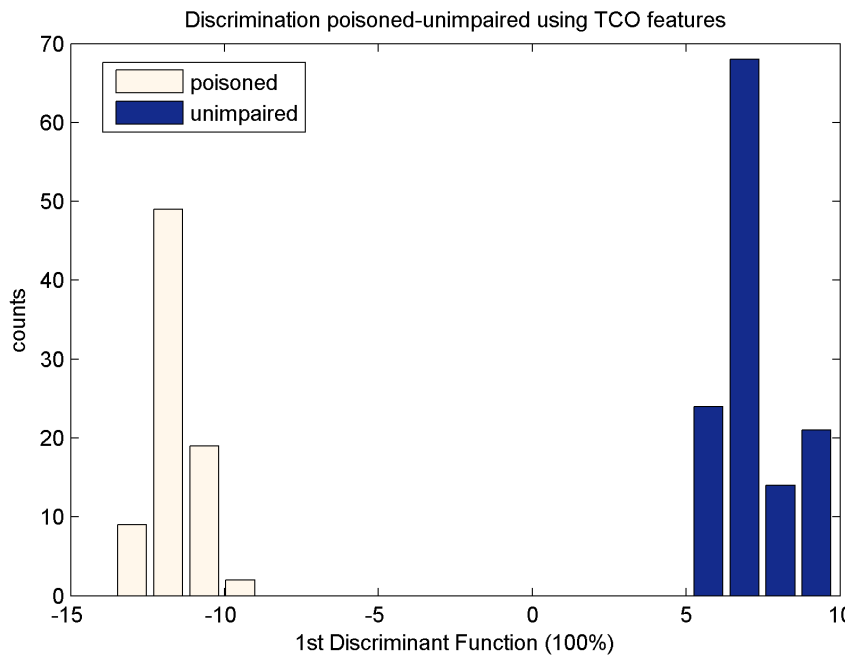

Figure 12. LDA discriminating measurements carried out with the poisoned (bright bars) and unimpaired (dark bars) sensor using five TCO features (absolute impedance mean value differences). The data were acquired using the FoBIS impedance spectroscope; measured conditions are pure air plus two concentrations each of $\mathrm{CH}_{4}$ $(550,1100 \mathrm{ppm})$ and $\mathrm{CO}(50,100 \mathrm{ppm})$, all at $50 \% \mathrm{rh}$.

To extract the features for the TCO-based LDAs shown in Figs. 10 and 11, we calculated the differences of the normalized absolute impedance values at $61 \mathrm{kHz}$ at adjacent temperature steps, i.e.,

$$
\begin{aligned}
& \left|Z\left(61 \mathrm{kHz}, T_{i}\right)\right|-\left|Z\left(61 \mathrm{kHz}, T_{i-1}\right)\right| \\
& i=1 \ldots 5, T_{0}=200{ }^{\circ} \mathrm{C}, T_{1}=250{ }^{\circ} \mathrm{C}, T_{2}=300^{\circ} \mathrm{C}, \\
& T_{3}=350{ }^{\circ} \mathrm{C}, T_{4}=400^{\circ} \mathrm{C}, T_{5}=450{ }^{\circ} \mathrm{C} .
\end{aligned}
$$


Again, 10 measurements were averaged for each FoBISbased data point.

Figure 10 shows the TCO-IA LDA: for the unimpaired sensor, the different gases are well separated, allowing a perfect classification. For the poisoned sensor, the LDA yields results which have strongly shifted. Unlike the EIS-based LDA, these shifts are of similar direction and value for the different gas exposures. This result would not enable a correct classification of either gas. In the TCO-FoBIS LDA (Fig. 11), the poisoning causes significant shifts as well, although the cross-validation shows a partially correct classification even after poisoning. Thus, a check of this result, which takes into account the EIS-based result (as outlined in Fig. 1), can increase the reliability in the detection of sensor changes.

Figure 12 shows the result of a TCO-based LDA carried out with data from all measurements shown in Fig. 11, but separated into two different classes: poisoned sensor and unimpaired sensor. The result shows that a detection of sensor impairments is also possible from TCO data acquired using the FoBIS impedance spectroscope for the limited test gas selection that was examined.

\section{Conclusion and outlook}

We developed a compact high-speed impedance measurement system, which enables the combination of EIS and TCO even for sensors with small thermal time constants, i.e., MEMS-based sensors. Sensor changes affect the results of both measurement methods differently, which enables a reliable detection of sensor impairments for improved reliability of gas sensor systems. The shifts in the EIS-based LDAs suggest that the impedance data contain information useful for recognition of sensor impairments. The long measurement time of the commercial impedance analyzer is an important challenge in temperature-cycled operation, which shows the necessity of faster impedance measurement systems for combined TCO-EIS measurements. The evaluation of TCO features indicates that a detection of sensor impairments can be possible from TCO data only. Therefore, further research will consider the influence of sensor changes to the dynamic behavior of the sensors and also examine the influence of various impairments (e.g., sulfur compounds), as well as broader ranges of test gases and gas concentrations.

Acknowledgements. We gratefully acknowledge funding by the German ministry of Economic Affairs and Energy (BMWi, grant no. $16962 \mathrm{~N})$. The authors would like to thank $3 \mathrm{~S} \mathrm{GmbH}$, Saarbrücken, Germany, for hardware support.

Edited by: M. Meyyappan

Reviewed by: one anonymous referee

\section{References}

Agilent 4294A: Precision Impedance Analyzer Data Sheet, available at: http://cp.literature.agilent.com/litweb/pdf/5968-3809E. pdf, last access: 25 November 2013.

Backhaus, K., Erichson, B., Plinke, W., and Weiber, R.: Multivariate Analysemethoden, Springer-Verlag, ISBN 3540-67146-3, 2000.

Barsoukov, E. and Macdonald, J. R.: Impedance Spectroscopy Theory, Experiment, and Application, John Wiley \& Sons, ISBN 0-471-64749-7, 2005.

Bârsan, N. and Weimar, U.: Understanding the fundamental principles of metal oxide based gas sensors; the example of CO sensing in the presence of humidity, J. Phys. Condens. Matter, 15, R813R839, 2003.

Clifford, P. K. and Tuma, D. T.: Characteristics of semiconductor gas sensors II. Transient response to temperature change, Sensors Actuators, 3, 255-281, 1982, 1983.

Conrad, T., Trümper, F., Hettrich, H., and Schütze, A.: Improving the Performance of Gas Sensor Systems by Impedance Spectroscopy: Application in Under-Ground Early Fire Detection, Proc. SENSOR Conference 2007, Volume I, AMA Service GmbH, ISBN 978-3-9810993-1-7, 169-174, 2007.

Elmi, I., Zamponelli, S., Cozzani, E., Mancarella, F., and Cardinali, G. C.: Development of ultra-low-power consumption MOX sensors with ppb-level VOC detection capabilities for emerging applications, Sensors and Actuators B, 135, 342-351, 2008.

Francois, D., Wertz, V., and Verleysen, M.: Choosing the Metric: A Simple Model Approach, Meta-Learning in Computational Intelligence Studies in Computational Intelligence, 358, 97-115, 2011.

Fricke, T., Reimann, P., Horras, S., Leonhardt, E., Sahm, P., Schütze, A.: A systematic approach for the automatic signal processing, evaluation and optimization of T-cycled gas sensors, IEEE International Instrumentation and Measurement Technology Conference $\mathrm{I}^{2}$ MTC, 2008.

Heilig, A., Bârsan, N., Weimar, U., Schweizer-Berberich, M., Gardner, J. W., and Göpel, W.: Gas identification by modulating temperatures of $\mathrm{SnO}_{2}$-based thick film sensors, Sensors and Actuators B, 43, 45-51, 1997.

Kohl, D.: Surface processes in the detection of reducing gases with SnO2-based devices, Sensors Actuators, 18, 71-113, 1989.

Korotchenkov, G. and Cho, B. K.: Instability of metal oxide-based conductometric gas sensors and approaches to stability improvement (short survey), Sensors and Actuators B, 156, 527-538, 2011.

Lee, A. P. and Reedy, B. J.: Temperature modulation in semiconductor gas sensing, Sensors and Actuators B, 60, 35-42, 1999.

Ljung, L. and Glad, T.: Modeling of dynamic systems, PTR Prentice-Hall, Upper Saddle River, NJ, USA, 1994.

Luo, D., Ding, C., and Huang, H.: Linear Discriminant Analysis: New Formulations and Overfit Analysis, Proceedings of the 25th AAAI Conference on Artificial Intelligence, 2011.

Morrison, S. R.: Semiconductor gas sensors, Sensors Actuators, 2, 329-341, 1982.

Morrison, S. R.: Selectivity in semiconductor gas sensors, Sensors Actuators, 12, 425-440, 1987.

Reimann, P.: Gasmesssysteme basierend auf Halbleitergassensoren für sicherheitskritische Anwendungen mit dem Ansatz der Sen- 
sorselbstüberwachung, Dissertation, Shaker Verlag, ISBN 9783-8440-0232-4, 2011.

Reimann, P., Dausend, A., and Schütze, A.: A self-monitoring and self-diagnosis strategy for semiconductor gas sensor systems, Proc. IEEE Sensors Conference 2008, Lecce, Italy, 27-29 October, 2008.

Schüler, M., Sauerwald, T., Walter, J., and Schütze, A.: High speed impedance spectroscope for metal oxide gas sensors, Lecture Notes on Impedance Spectroscopy, 5, accepted, 2014.

Schütze, A., Gramm, A., and Rühl, T.: Identification of Organic Solvents by a Virtual Multisensor System with Hierarchical Classification, IEEE Sensors, 4, 857-863, 2004.
Stetter, J. R. and Penrose, W. R.: Understanding Chemical Sensors and Chemical Sensor Arrays (Electronic Noses): Past, Present, and Future, Sensors update 10.1, 10, 189-229, 2002.

Tricoli, A., Righettoni, M., and Teleki, A.: Semiconductor gas sensors: dry synthesis and application, Angew. Chem. Int. Ed., 49, 7632-7659, doi:10.1002/anie.200903801, 2010.

Weimar, U. and Göpel, W.: A.C. measurements on tin oxide sensors to improve selectivities and sensitivities, Sensors and Actuators B, 26, 13-18, 1995. 